\title{
A CSF3R T618I Mutation in a Patient with Chronic Neutrophilic Leukemia and Severe Bleeding Complications
}

\author{
Toru Mitsumori ${ }^{1}$, Norio Komatsu ${ }^{2}$ and Keita Kirito ${ }^{1}$
}

\begin{abstract}
Chronic neutrophilic leukemia (CNL) is a rare form of myeloproliferative neoplasm characterized by the drastic elevation of mature neutrophils. One of the major causes of death among patients with CNL is severe bleeding; however, the difficulty of accurately diagnosing this disease has caused confusion in this field. Recently, somatic mutations of the CSF3R gene have been associated with CNL. This has led to the establishment of more accurate diagnostic criteria for CNL. We herein report a case study of a patient with CNL with a T618I point mutation on the CSF3R gene who showed severe bleeding.
\end{abstract}

Key words: CNL, CSF3R mutation, bleeding tendency

(Intern Med 55: 405-407, 2016)

(DOI: 10.2169/internalmedicine.55.5059)

\section{Introduction}

Chronic neutrophilic leukemia (CNL) is a rare myeloproliferative neoplasm characterized by an extreme increase in the number of mature neutrophils (1). According to a review by Gotlib et al., approximately 200 cases of CNL have been reported to date (2). Because of the lack of useful and specific clonal markers for CNL, the WHO 2008 diagnostic criteria for $\mathrm{CNL}$ are highly dependent on the exclusion of other types of myeloid malignancies and reactive neutrophilia (3). This makes the diagnosis of CNL challenging. In fact, Pardanani et al. stated that among 35 suspected cases of CNL, only 12 cases actually met the WHO-defined diagnostic criteria for CNL (4).

In 2013, Maxson et al. identified an oncogenic mutation in the colony-stimulating factor 3 receptor $(C S F 3 R)$ gene, which encodes the G-CSF receptor in CNL and atypical chronic myeloid leukemia (aCML). This condition is a variant of the myelodysplastic/myeloproliferative neoplasms (MDS/MPN) (5). The identification of the CSF3R mutation in CNL greatly improves the diagnosis of this disease (3).

Two primary types of CSF3R mutations have been identified to date: truncation mutations and membrane proximal mutations (including the T618I mutation) (5). Pardanani et al. demonstrated that all of the patients with CNL who were diagnosed according to the WHO 2008 diagnostic criteria for CNL had the T618I mutation; in contrast, none of the patients with aCML had this mutation (4). Thus, the CSF3R T618I mutation is highly restricted to CNL and is likely to be a specific marker for this disease (3).

One of the most serious complications of CNL is increased bleeding, which occasionally results in death; however, the pathological mechanism of this phenomenon is not fully understood.

Here, we present the case of a patient with T618I-positive $\mathrm{CNL}$ and severe bleeding. The patient's platelet function was not impaired, which suggests that factors other than platelet dysfunction contribute to the tendency for bleeding in patients who are accurately diagnosed with CNL.

\section{Case Report}

A 77-year-old man was admitted to our hospital with bleeding and an increased white blood cell count. He had splenomegaly and hepatomegaly but not lymphadenopathy. Massive subcutaneous hemorrhages were found in his upper limbs and chest (Fig. 1A), and his peripheral blood cell counts revealed a marked elevation of white blood cells $\left(150.0 \times 10^{9} \quad\right.$ cells $/ \mathrm{L}$; normal value: $\left.3.6-9.7 \times 10^{9} / \mathrm{L}\right)$, which

${ }^{1}$ Department of Hematology and Oncology, University of Yamanashi, Japan and ${ }^{2}$ Department of Hematology, Juntendo University School of Medicine, Japan

Received for publication January 30, 2015; Accepted for publication May 24, 2015

Correspondence to Dr. Keita Kirito, kirito@yamanashi.ac.jp 

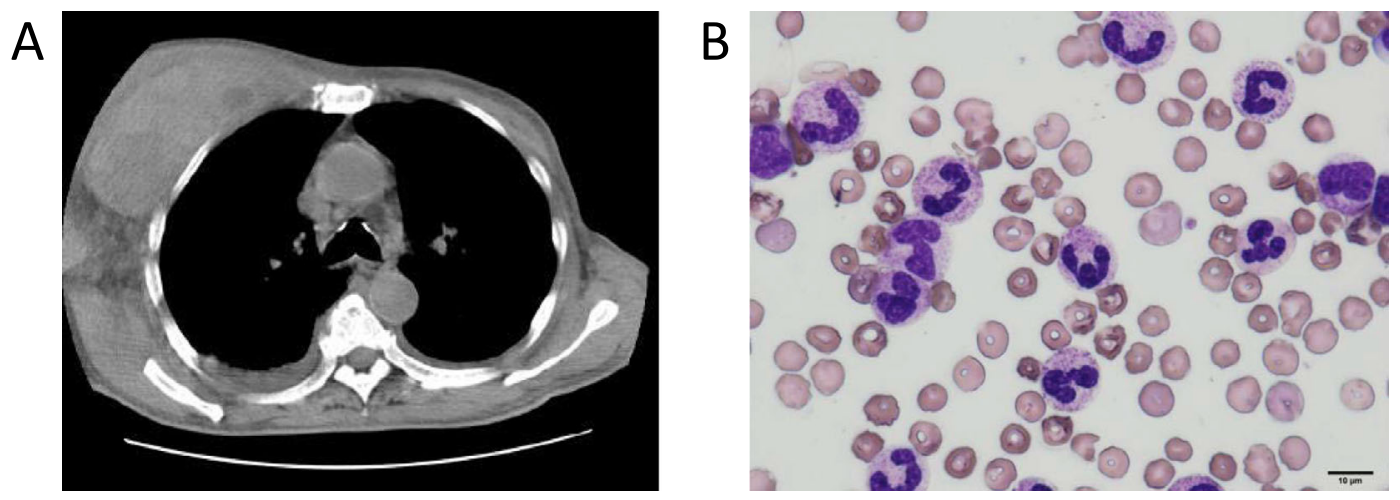

Figure 1. (A) A CT scan showing a massive subcutaneous hemorrhage in the thorax. (B) A peripheral blood smear showing an increase in the number of mature neutrophils with toxic granules.

control

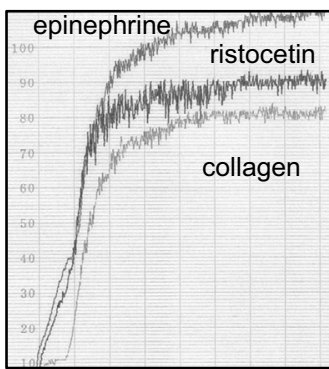

Patient

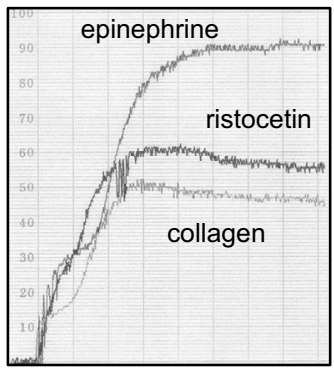

control

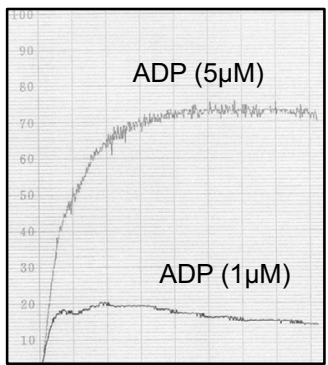

Patient

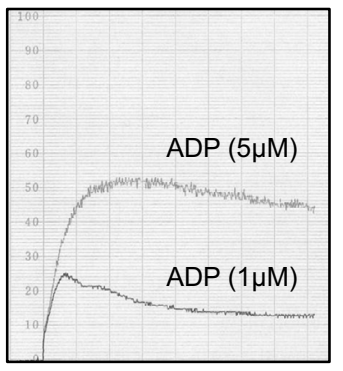

Figure 2. A platelet aggregation test. Left panel: normal control; right panel: the patient. Platelet aggregation was triggered by epinephrine $(2 \mu \mathrm{M})$, ristocetin $(1.25 \mathrm{mg} / \mathrm{mL})$, collagen $(2 \mu \mathrm{g} / \mathrm{mL})$ and ADP (1 or $5 \mu \mathrm{M})$.

were primarily composed of mature neutrophils (promyelocytes $2 \%$, myelocytes $9 \%$, meta-myelocytes $4 \%$, neutrophils $82 \%$ ) with toxic granules (Fig. 1B). The patient also showed moderate anemia ( $\mathrm{Hb} 8.0 \mathrm{~g} / \mathrm{dL}$; normal values 11.1-14.8 g/ $\mathrm{dL}$ ) and thrombocytopenia (platelet count $120 \times 10^{9} / \mathrm{L}$; normal values 155 to $348 \times 10^{9} / \mathrm{L}$ ). The hemostatic tests demonstrated the following values: bleeding time, 3 minutes (normal range: 1-5 minutes), prothrombin time, $12.8 \mathrm{~s}$ (normal range: 9.5-13.1 s), activated partial thromboplastin time, $37.8 \mathrm{~s}$ (normal range: 29.7-47.4 s) and fibrinogen, $263 \mathrm{mg} /$ dL (normal range: $150-330 \mathrm{mg} / \mathrm{dL}$ ). The patient's factor VIII and factor IX activities were $133 \%$ and $103 \%$, respectively. To investigate the cause of the increased bleeding, we analyzed the patient's platelet function (Fig. 2). The platelet aggregation in response to epinephrine, ristocetin and ADP (1 $\mu \mathrm{M}$ ) was not impaired. The response to collagen or ADP (5 $\mu \mathrm{M})$ was modestly decreased in comparison to normal patients.

A fluorescence in situ hybridization (FISH) study revealed that the patient did not have the $B C R-A B L$ fusion gene, and no monoclonal gammopathy was detected. After approval was obtained from the institutional review board of the University of Yamanashi and the patient provided written informed consent, we prepared DNA from the peripheral blood neutrophils and analyzed the mutation status of JAK2. The informed consent document included the provision of consent for a DNA analysis using residual DNA for a future study. We did not find a $J A K 2$ V617F mutation. Unfortunately, the patient refused to undergo a bone marrow biopsy.

We treated the patient using hydroxyurea and a blood transfusion; however, his white blood cell count subsequently became markedly elevated and he died due to multiple organ failure within 6 months of his initial presentation. After the report from Maxson et al., which showed the presence of the CSF3R mutation in patients with CNL (5), we tested whether the CSF3R mutation was present in our patient using residual DNA. As Fig. 3 shows, we identified a C-to-T transition in exon 4 of the CSF3R gene, which was identical to the T618I point mutation.

\section{Discussion}

This case report is the first to confirm the presence of the CSF3R T618I mutation in a patient with CNL presenting with a severe bleeding tendency. Patients with CNL frequently exhibit a severe bleeding tendency (6). A review of by Elliott found intracranial hemorrhages in 9 of $40 \mathrm{CNL}$ patients (6). In addition, a series of case reports demonstrated severe bleeding complications in CNL patients $(7,8)$, which suggests that CNL is correlated with increased bleeding; however, the precise pathological mechanism of this relationship remains unclear. Elliott suggested that the bleeding complications observed in patients with CNL are associated with treatment-induced thrombocytopenia (6); however, 


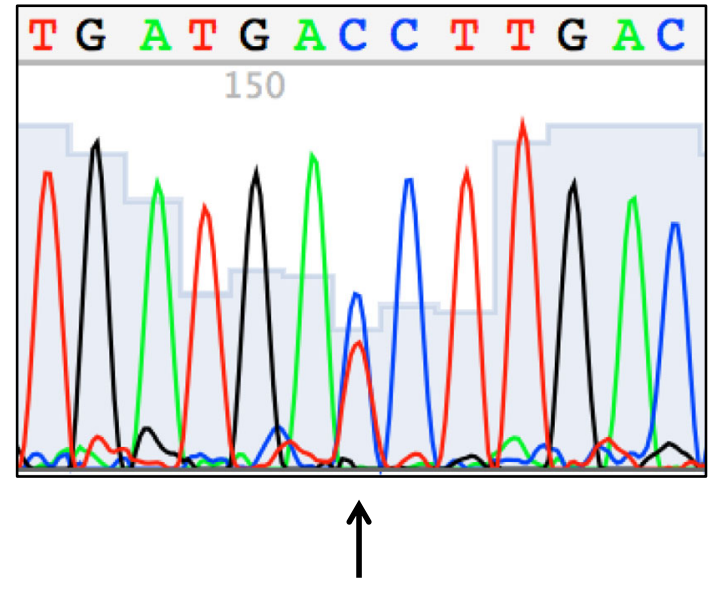

Figure 3. The direct sequencing of exon 14 of the CSF3R gene. The arrow indicates the $\mathrm{C}$-to- $\mathrm{T}$ mutation that resulted in T618I.

some case reports have indicated the presence of platelet dysfunction in these patients $(7,9-12)$. The majority of CNL patients presenting with platelet dysfunction were reported before the establishment of the WHO 2008 diagnostic criteria for CNL. It is therefore unclear whether these cases actually met the WHO 2008 criteria. Our present case was confirmed to have the $C S F 3 R$ T618I mutation, a highly specific molecular marker for CNL (3). This finding further supports the CNL diagnosis of this patient. Although our patient showed a severe bleeding tendency at diagnosis, he also showed a normal bleeding time, and his platelet number was only modestly decreased. In addition, we confirmed that his platelet aggregation was not impaired. These results suggest that the bleeding tendency in accurately diagnosed patients with CNL does not occur due to a lower platelet count or impaired platelet function. Although we did not perform a histological examination, it is also possible that the increase in neutrophil numbers led to the invasion and destruction of the vascular wall (13). Interestingly, mice transplanted with CSF3R T618I-expressing bone marrow cells showed the infiltration of mature neutrophils in multiple organs (14). Taken together, a T618I mutation might confer a proliferative advantage and increase the invasion ability of neutrophils.

We treated the patient with hydroxyurea; however, the bleeding tendency of the patient did not improve. Interestingly, several recent studies have demonstrated that the JAK1/2 inhibitor ruxolitinib is potentially useful in the treatment of patients with CNL with the CSF3R T618I mutation. The administration of ruxolitinib decreased the white blood cell counts and led to the recovery of platelets in CSF3RT618I-mutation-positive patients with $\operatorname{CNL}(5,15)$. This drug also improved the patients' massive splenomegaly and constitutional symptoms (15). Although the hypothesis is only speculative at present, if the bleeding tendency shown in patients with CNL were caused by the invasion of malignant neutrophils, then ruxolitinib might also improve the bleeding tendency in CSF3R-T618I-positive patients with CNL.

Additional studies involving T618I-positive CNL patients with a bleeding tendency are required to elucidate the mechanisms through which the lethal bleeding phenotype is manifested these patients and to establish a new treatment strategy.

The authors state that they have no Conflict of Interest (COI).

\section{References}

1. Zoi K, Cross NP. Molecular pathogenesis of atypical CML, CMML and MDS/MPN-unclassifiable. Int J Hematol 101: 229242, 2015.

2. Gotlib J, Maxson JE, George TI, Tyner JW. The new genetics of chronic neutrophilic leukemia and atypical CML: implications for diagnosis and treatment. Blood 122: 1707-1711, 2013.

3. Tefferi A, Thiele J, Vannucchi AM, Barbui T. An overview on CALR and CSF3R mutations and a proposal for revision of WHO diagnostic criteria for myeloproliferative neoplasms. Leukemia $\mathbf{2 8}$ : 1407-1413, 2014.

4. Pardanani A, Lasho TL, Laborde RR, et al. CSF3R T618I is a highly prevalent and specific mutation in chronic neutrophilic leukemia. Leukemia 27: 1870-1873, 2013.

5. Maxson JE, Gotlib J, Pollyea DA, et al. Oncogenic CSF3R mutations in chronic neutrophilic leukemia and atypical CML. N Engl J Med 368: 1781-1790, 2013.

6. Elliott Md MA. Chronic neutrophilic leukemia and chronic myelomonocytic leukemia: WHO defined. Best Pract Res Clin Haematol 19: 571-593, 2006

7. Shigekiyo T, Miyagi J, Chohraku M, et al. Bleeding tendency in chronic neutrophilic leukemia. Int J Hematol 88: 240-242, 2008.

8. Imashuku S, Kudo N, Kubo K, Saigo K, Okuno N, Tohyama K. Rituximab for managing acquired hemophilia $\mathrm{A}$ in a case of chronic neutrophilic leukemia with the JAK2 kinase V617F mutation. J Blood Med 3: 157-161, 2012.

9. Mehrotra PK, Winfield DA, Fergusson LH. Cellular abnormalities and reduced colony-forming cells in chronic neutrophilic leukaemia. Acta Haematol 73: 47-50, 1985.

10. Hossfeld DK, Lokhorst HW, Garbrecht M. Neutrophilic leukemia accompanied by hemorrhagic diathesis: Report of two cases. Blut 54: 109-113, 1987.

11. Lorente JA, Peña JM, Ferro T, et al. A case of chronic neutrophilic leukemia with original chromosomal abnormalities. Eur $\mathbf{J}$ Haematol 41: 285-288, 1988.

12. Zittoun R, Réa D, Ngoc LH, Ramond S. Chronic neutrophilic leukemia. Ann Hematol 68: 55-60, 1994.

13. Noguchi $T$, Ikeda $K$, Yamamoto $K$, et al. Severe bleeding tendency caused by leukemic infiltration and destruction of vascular walls in chronic neutrophilic leukemia. Int J Hematol 74: 437-441, 2001.

14. Fleischman AG, Maxson JE, Luty SB, et al. The CSF3R T618I mutation causes a lethal neutrophilic neoplasia in mice that is responsive to therapeutic JAK inhibition. Blood 122: 3628-3631, 2013.

15. Dao K-HT, Solti MB, Maxson JE, et al. Significant clinical response to JAK1/2 inhibition in a patient with CSF3R-T618Ipositive atypical chronic myeloid leukemia. Leuk Res Rep 3: 6769, 2014.

(C) 2016 The Japanese Society of Internal Medicine http://www.naika.or.jp/imonline/index.html 